\section{POLYUNSATURATED FATTY ACIDS (PUFAS) AND SPECIALIZED PRO-RESOLVING MEDIATORS (SPMS) ARE DECREASED IN PLASMA AND SERUM FROM SLE PATIENTS COMPARED TO HEALTHY CONTROLS}

${ }^{1}$ Julia Davis-Porada*, ${ }^{2}$ Charles Serhan, ${ }^{2}$ Paul Norris, ${ }^{3}$ Peter Lipsky, ${ }^{4}$ Jane Salmon. ${ }^{1}$ Hospital for Special Surgery; ${ }^{2}$ Center for Experimental Therapeutics and Reperfusion Injury Department of Anesthesia, Perioperative and Pain Medicine Brigham and Womens Hospital and Harvard Medical School; ${ }^{3}$ AMPEL BioSolutions; ${ }^{4}$ Hospital for Special Surgery, Weill Cornell Medicine

\subsection{6/lupus-2019-Ism.3}

Background Systemic lupus erythematosus (SLE) is an autoimmune disease with persistent, inflammatory mediated organ damage. It has been suggested that omega-3-polyunsaturated fatty acids (PUFAs) are low in SLE patients and that supplementation with omega-3 PUFAs might be beneficial. Omega-3 PUFAs can be metabolized to specialized pro-resolving mediators (SPM) in inflamed tissues. PUFAs, eicosapentaenoic acid (EPA) and docosahexaenoic acid (DHA), give rise to protectins and E-series and d-series resolvins, respectively. These SPMs help promote tissue repair and healing in addition to reducing neutrophil infiltration. We sought to determine whether EHA and DHA and SPMs were dysregulated in lupus patients compared to controls.

Methods Blood samples were collected from 12 patients enrolled in the Autoimmune Disease Registry and Repository, a single center registry (1996-present) of patients meeting ACR SLE classification criteria. Samples were collected from 12 non-SLE-controls who were age ( \pm 5 years) and race/ethnicity matched. Metabolomic profiling via tandem mass spectrometry (LC-MS-MS) was performed on serum and plasma to assess the PUFA and SPM levels.

Results Levels of EPA and DHA were highly correlated in serum and plasma. Both EPA and DHA were significantly decreased in SLE patients compared to controls (table 1). Neither plasma nor serum DHA or EPA levels was correlated with disease activity assessed by SLEDAI score. SPMs including PD1 and RvE1 as well as their precursors, 17-HDHA and 18-HEPE, were identified in plasma and serum samples from SLE patients. Plasma levels of 17-HDHA, as well as serum levels of PD1, 17-HDHA, and 18-HEPE tended to be reduced in SLE (table 1). The SLE patients with a history of nephritis had significantly lower levels of DHA $(p=0.03)$, EPA $(p=0.05)$, 18-HEPE $(p=0.03)$, and 17-HDHA $(p=0.04)$ than SLE patients without nephritis.

Conclusions SLE patients have lower levels of circulating EPA and DHA, the substrates for SPMs, relative to individuals without SLE. Lower levels of these PUFAs and some SPMs are associated with history of nephritis. Additionally, the levels of PD1, 17-HDHA, and 18-HEPE were measurable in SLE serum and plasma and tended to be reduced, especially in subjects with lupus nephritis. SPMs suppress the production of inflammatory mediators and promote resolution of inflammation. The lower levels of PUFAs and SPMs could contribute to the likelihood of developing lupus nephritis. Further evaluation of this relationship is warranted.

\section{$4 \quad$ ANTI-RETINOBLASTOMA PROTEIN ANTIBODIES ARE NEGATIVELY ASSOCIATED WITH LUPUS NEPHRITIS}

Jessica Li, Andreas Goules, Daniel Goldman, Antony Rosen, Livia Casciola-Rosen, Michelle Petri* . Johns Hopkins University School of Medicine

\subsection{6/lupus-2019-Ism.4}

Background Retinoblastoma protein (RB) regulates nucleosome/chromatin structures and is linked to tumor suppression. It regulates the cell cycle by repression of E2F transcription factor and stabilization of heterochromatin. Because SLE is the prototypic autoimmune disease with autoantibodies against the nucleosome and chromatin, the presence of anti-RB antibodies and the association with disease manifestations were examined.

Methods 222 SLE patients from the Hopkins longitudinal cohort seen consecutively in clinic were studied $(85 \%$ female, 94\% Caucasian, mean age 51 years). Anti-RB antibodies were assayed by immunoprecipitation of 35S-methionine-labeled protein generated by in vitro transcription and translation from full length human cDNA. Odds ratios and p-values for univariate analyses were calculated using Fishers exact t-test. Exact logistic regression and odds ratios were calculated for the multi-variate model due to a cell frequency of zero for proteinuria ever and positive anti-RB antibody status.

Results Anti-RB antibodies were present in $8.6 \%$ of these SLE patients, $6.3 \%$ with medium-high titer. Univariate associations with SLE manifestations for the medium-high titer positive patients are included in table 1 . Of note, medium/high titer anti-RB antibodies were never found in patients with proteinuria $(p=0.0028)$. In a multi-variate model for proteinuria, anti$\mathrm{RB}$ antibodies remained negatively associated (OR 0.1112, $\mathrm{p}=0.016$ ) after correction for female gender (OR 0.417, $\mathrm{p}=0.0498$ ), ethnicity (Caucasian, OR 0.288, $\mathrm{p}=0.0833$ ), low anti-dsDNA ever (OR 1.806, $\mathrm{p}=0.1192$ ), anti-Sm positive ever (OR 1.273, $\mathrm{p}=0.7127)$ and low complement ever (OR 2.111, $\mathrm{p}=0.0377)$.

\begin{tabular}{|c|c|c|c|c|}
\hline & $\begin{array}{l}\text { Metabolite (in serum) } \\
\text { mean, (SD) }\end{array}$ & Control (n=12) & SLE (n=12) & P Value \\
\hline & EPA & $6779.7(3985.2)$ & 2822.9 (3082.9) & 0.013 \\
\hline & DHA & $19584.0(15785.1)$ & 7664.0 (5613.3) & 0.003 \\
\hline & PD1 & $55.0(128.6)$ & $23.3(57.1)$ & 0.489 \\
\hline & 17-HDHA & $47.4(25.3)$ & $15.7(14.9)$ & $<0.001$ \\
\hline & 18-HEPE & $20.8(7.1)$ & $17.3(9.8)$ & 0.195 \\
\hline
\end{tabular}

